\title{
Design and test of an EMCCD CCD201 sensor driving circuit
}

\author{
Qiang $\mathrm{Wu}^{\mathrm{a}}$, Zhao-Hui Feng ${ }^{\mathrm{b}}$ and $\mathrm{Xu}-\mathrm{Wen} \mathrm{Li}^{\mathrm{c}}$ \\ Beijing University of Technology, Beijing, China \\ E-mail: ${ }^{a}$ wuqiang@bjut.edu.cn, ${ }^{b} f z h 165 @ 163 . c o m,{ }^{c}$ lixuwen@bjut.edu.cn \\ www.bjut.edu.cn
}

\begin{abstract}
In order to improve the imaging quality in extremely low illumination situation, a driving circuit of high resolution and sensitivity camera was developed to use back illumination. Frame transfer electron multiplying gains (EMCCD) with $1024 \times 1024$ pixels named CCD201 from UK E2V technologies. The circuit uses integrated CMOS driver EL7457 and EL7156 as the driving module. A programmable control voltage power source was adopted for the electron multiplying voltage to realize program-EM control. A bias voltage circuit with a high efficiency and precision was designed for the CCD201 complex voltage supply system.
\end{abstract}

Keywords: EMCCD; CCD201; Driving Circuit; Bias Voltage.

\section{Introduction}

A Gain register set between readout register and output amplifier in EMCCD chip enlarge CCD light raw charge signal by mechanism of charge avalanche multiplication gain silicon. Compared with traditional CCD, it has many advantages of small volume, low noise, high sensitivity, strong detection capability; it has attracted much attention in the field of military and civilian applications. Such as low light level image acquisition or space exploration, technology of EMCCD occupies a leading and dominant position. EMCCD is sensitive to any wavelength range of light, it makes the camera system which based on EMCCD has excellent performance [1].

CCD201 is EMCCD sensor which signal charge can be linearly amplified 1000 times. It is capable of detecting task in extremely low illumination situation $\left(10^{-3} \mathrm{Lux}\right)$. This paper introduces methods about CCD201 driving and bias voltage circuit which is the key technology of EMCCD camera.

\section{Operational Principle of EMCCD}

\subsection{Operational principle of EMCCD}


The biggest difference between the traditional CCD and EMCCD is the quality of imaging in the low light level. It mainly benefit from the structure and strict manufacturing processes [2]. Figure.1 shows the physical structure of CCD201 sensor. Frame transfer CCDs consist of two areas: an image area, which captures the image, and a storage area, where the image is stored prior to read out. The image and store sections are designed to operate in 2-phase mode, to maximize the highest achievable transfer frequency. Following transfer through the readout register, the signal charge is multiplied in the gain register before conversion to a voltage by an output amplifier.

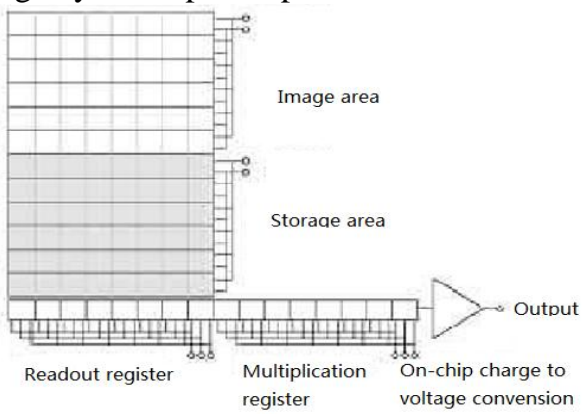

Fig. 1 Schematic diagram of an EMCCD

\subsection{Introduction of EMCCD Chip}

The chosen EMCCD chip is CCD201, which is produced by E2V Company. CCD201 is a back illuminated sensor, which has maximal resolution at present, the pixel size is $13 \times 13 \mu \mathrm{m} 2$, its resolution is 1024 (horizontal) $\times 1024$ (vertical), and it has $100 \%$ filling rate. This device uses a novel output amplifier circuit that is capable of operating at an equivalent output noise of less than one electron at pixel rates of over $15 \mathrm{MHz}$. This makes the sensor well suited for scientific imaging where the illumination is limited.

Tab. 1 shows the differences between previous generation products CCD97 and latest product CCD201 chip.

Tab. 1 General data between CCD97 and CCD201

\begin{tabular}{ccc}
\hline Parameter Data & CCD97 & CCD201 \\
\hline Active pixels & $512(\mathrm{H}) \times 512(\mathrm{~V})$ & $1024(\mathrm{H}) \times 1024(\mathrm{~V})$ \\
Image pixel size & $16 \times 16[\mu \mathrm{m}]$ & $13 \times 13[\mu \mathrm{m}]$ \\
Pixel rate & $15[\mathrm{MHz}]$ & $20[\mathrm{MHz}]$ \\
Power dissipation & $\leq 1500[\mathrm{~mW}]$ & $\leq 2000[\mathrm{~mW}]$ \\
Active image area & $8.192 \times 8.192[\mathrm{~mm}]$ & $13.3 \times 13.3[\mathrm{~mm}]$ \\
Dark signal $(293 \mathrm{~K})$ & $800[\mathrm{e}-/ \mathrm{pixel} / \mathrm{s}]$ & $530[\mathrm{e}-/ \mathrm{pixel} / \mathrm{s}]$ \\
\hline
\end{tabular}


As shown in the table, CCD201 has the larger active image and higher resolution. In the meantime, the system which equipped with CCD201 has higher frame rate and short sampling period.

\section{Design of CCD201 Driving Circuit}

\subsection{Requirement analysis of CCD201 driving circuit}

CCD201 sensor requires four imaging timing input, four store driving input, three register input, one reset signal and a program-EM signal. The program-EM is the most complex signal among these driving circuits. Table. 2 shows the different voltages among the driving circuit.

Tab. 2 Table of driving voltage

\begin{tabular}{|c|c|c|c|}
\hline \multirow{2}{*}{ signal } & \multicolumn{2}{|c|}{ voltage } & \multirow{2}{*}{ capacitance } \\
\hline & typical & range & \\
\hline I $\varnothing 1 / 2 / 3 / 4$ high & $7[\mathrm{~V}]$ & $5 \sim 9[\mathrm{~V}]$ & $22[\mathrm{nF}]$ \\
\hline $\mathrm{I} \emptyset 1 / 2 / 3 / 4$ low & $-5[\mathrm{~V}]$ & $-6 \sim-4[\mathrm{~V}]$ & $22[\mathrm{nF}]$ \\
\hline $\mathrm{S} \varnothing 1 / 2 / 3 / 4$ high & $7[\mathrm{~V}]$ & $5 \sim 9[\mathrm{~V}]$ & $17[\mathrm{nF}]$ \\
\hline $\mathrm{S} \emptyset 1 / 2 / 3 / 4$ low & $-5[\mathrm{~V}]$ & $-6 \sim-4[\mathrm{~V}]$ & $17[\mathrm{nF}]$ \\
\hline $\mathrm{R} \emptyset 1 / 2 / 3$ high & $12[\mathrm{~V}]$ & $8 \sim 13[\mathrm{~V}]$ & $180[\mathrm{pF}]$ \\
\hline $\mathrm{R} \emptyset 1 / 2 / 3$ low & $0[\mathrm{~V}]$ & - & - \\
\hline$\varnothing \mathrm{R}$ high & $10[\mathrm{~V}]$ & $8 \sim 12[\mathrm{~V}]$ & $180[\mathrm{pF}]$ \\
\hline$\emptyset \mathrm{R}$ low & $0[\mathrm{~V}]$ & - & - \\
\hline Rø2HV high & - & $36 \sim 46[\mathrm{~V}]$ & - \\
\hline $\mathrm{R} \emptyset 2 \mathrm{HV}$ low & $4[\mathrm{~V}]$ & - & - \\
\hline
\end{tabular}

Besides, other 9 different bais voltages is also needed to ensure CCD201 working properly, as shown in Tab. 3 .

Tab. 3 Table of bias voltages

\begin{tabular}{cccc}
\hline \multirow{2}{*}{ power } & \multicolumn{2}{c}{ voltage } & description \\
\hline Rypical & $3[\mathrm{~V}]$ & $2 \sim 5[\mathrm{~V}]$ & Multiplication Register DC \\
OG & $3[\mathrm{~V}]$ & $1 \sim 5[\mathrm{~V}]$ & Bias \\
IG & $-5[\mathrm{~V}]$ & - & Output Gate \\
SS & $4.5[\mathrm{~V}]$ & $0 \sim 7[\mathrm{~V}]$ & Isolation Gate \\
ODL/ODH & $28[\mathrm{~V}]$ & $25 \sim 32[\mathrm{~V}]$ & Substrate \\
RD & $17[\mathrm{~V}]$ & $15 \sim 20[\mathrm{~V}]$ & Output Drain \\
ABD & $18[\mathrm{~V}]$ & $10 \sim 20[\mathrm{~V}]$ & Reset Drain \\
DD & $24[\mathrm{~V}]$ & $20 \sim 25[\mathrm{~V}]$ & Anti-Blooming Drain \\
\end{tabular}




\subsection{The design of time driving circuit}

To meet the requirements of driving circuit, this paper using integrated CMOS chip EL7457 and EL7156 to improve the load capacity of the driving circuit. At the meanwhile, the level-shift is completed over here $[3,4]$.

The EL7457 is a high speed, non-inverting, quad CMOS driver produced by INTERSIL Company. It is capable of running at clock rates up to $40 \mathrm{MHz}$ and features $2 \mathrm{~A}$ peak drive capability and a nominal on-resistance of just $3 \Omega$. The EL7457 is ideal for driving highly capacitive loads, such as storage and vertical clocks in CCD applications. The EL7156 is a high performance CMOS driver with 3-state. It is suited to many ATE and level-shifting applications. The 3.5A peak drive capability makes this part an excellent choice when driving high capacitance loads. Fig. 2 shows the schematic circuit of EL7457 and EL7156.

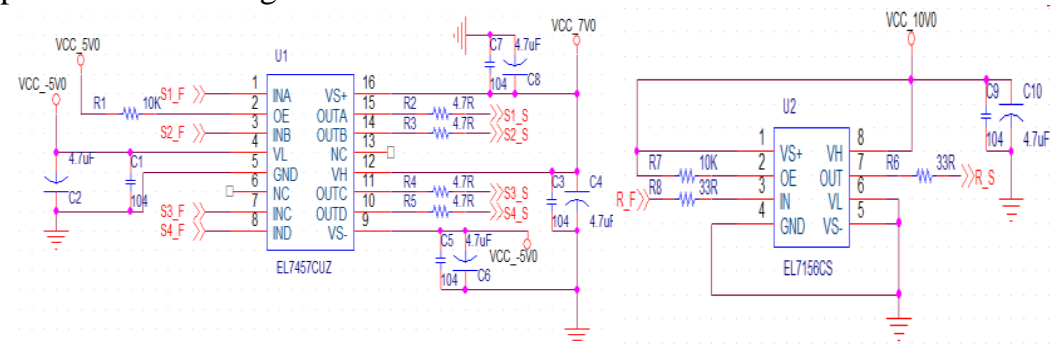

Fig. 2 Schematic circuit of EL7457

\subsection{The design of bias voltage circuit}

Fig. 3 shows the design diagram of bias voltage. The whole bias voltage system is supplied with single $12 \mathrm{~V}$ input, and then applied to different occasions. Using multistage cascade power supply circuit can provide a low noise, high efficiency power supply. As the figure. 3 shown, the primary voltage regulator is provided by $\mathrm{DC} / \mathrm{DC}$ convert, including negative bias voltage and positive bias voltage. And the secondary voltage regulator is composed of LDO regulator.

To meet the different bias voltage requirement, each bias voltage is powered by an independent power supply system. Compared with the single input voltage $12 \mathrm{~V}$, the smaller bias voltage such as $4.5 \mathrm{v}, 3 \mathrm{v}$ and $4 \mathrm{v}$, is provided by DC/DC+LDO buck circuit; the higher voltage $(17 \mathrm{v}, 18 \mathrm{v}, 24 \mathrm{v}, 28 \mathrm{v})$ is provided by "DC/DC+LDO" boost circuit. The negative bias voltage is directly generated by invert circuit. These multistage cascade bias voltage supply circuit ensure a low noise and high efficiency of the power supply system. 


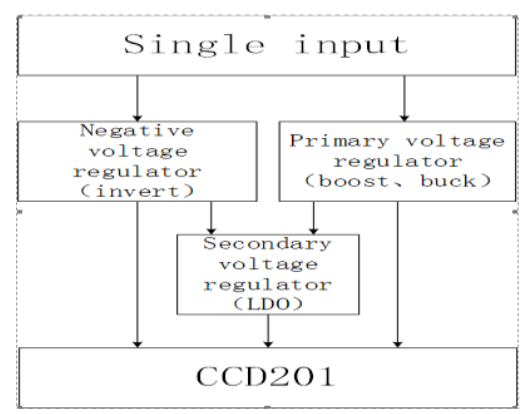

Fig. 3 Diagram of bias voltage

\subsection{The design of program-EM}

The key to implement multiplication gain technology is program-EM [5].According to data sheet of CCD201, the impact ionization effect will not be generated when R $\emptyset 2 \mathrm{HV}$ signal amplitude is lower than 20V. And the CCD201 will be damaged while R $\varnothing 2 \mathrm{HV}$ signal higher than $50 \mathrm{~V}[6,7]$.Aimed at such problems, this paper presents a particular solution. First of all, we divided this circuit into two parts: high voltage square wave generating circuit and voltage continuously adjustable circuit.

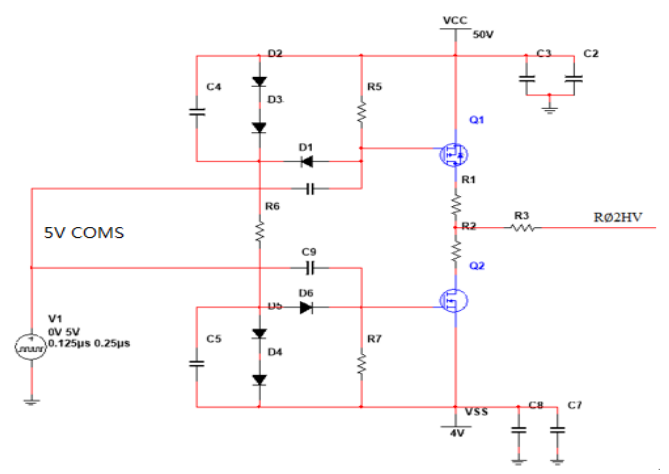

Fig. 4 Push-pull driving circuit

The high voltage square wave is realized by the MOSFET closing and opening alternately. But the Current provided from FPGA IO cannot provide load directly. There is a buffer circuit needed between FPGA and MOSFET. The following level conversion provided by a push-pull driving circuit, as shown in Figure.4. The design chooses BSS138P N-MOSFET and BSS84 P-MOSFET which is produced by NXP Semiconductors as the mainly components of circuit. The mainly reasons to choose MOSFET above are high-speed switching and high drain-source voltage $(>50 \mathrm{~V})$. 
Voltage continuously adjustable circuit is composed of a ratio phase amplifier and a voltage boost module. A boost DC/DC convert is needed to generate the high voltage level. And the final output voltage is controlled by adjusting the amplifier coefficient.

\section{The Test of Driving Circuit}

We created a CCD201 driving circuit board according to the design mentioned above. Figure. 5 shows the material object of CCD201 driver circuit board.

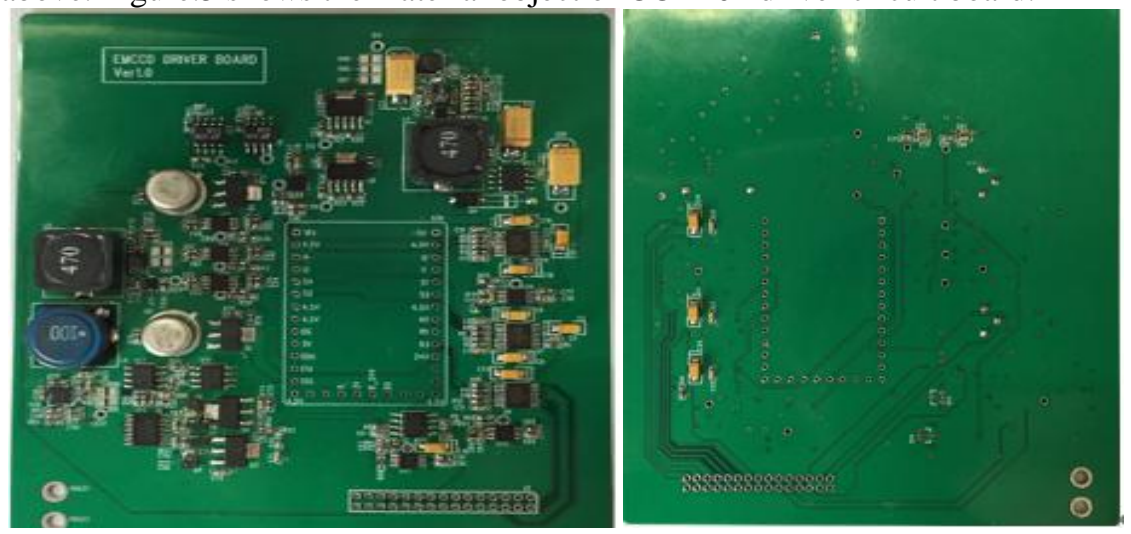

Fig. 5 Material object of CCD201 driver circuit board

As the table. 4 shown, the deviation between measured voltage and typical voltage is less than $2.5 \%$. It is suitable to ensure the CCD201 working stable.

Tab. 4 The results of the bias voltage tests

\begin{tabular}{ccc}
\hline signal & measured & typical \\
\hline I $\varnothing 1,2,3,4$ high & $7.03[\mathrm{~V}]$ & $7[\mathrm{~V}]$ \\
$\mathrm{I} \emptyset 1,2,3,4$ low\&IG & $-4.99[\mathrm{~V}]$ & $-5[\mathrm{~V}]$ \\
$\mathrm{R} \emptyset 1,2,3$ high\&DG high & $11.99[\mathrm{~V}]$ & $12[\mathrm{~V}]$ \\
R $\varnothing$ HV low & $3.99[\mathrm{~V}]$ & $4[\mathrm{~V}]$ \\
$\varnothing \mathrm{R}$ high & $10.15[\mathrm{~V}]$ & $10[\mathrm{~V}]$ \\
OG \&RøDC & $2.99[\mathrm{~V}]$ & $3[\mathrm{~V}]$ \\
SS & $4.45[\mathrm{~V}]$ & $4.5[\mathrm{~V}]$ \\
ODL/ODH & $27.37[\mathrm{~V}]$ & $28[\mathrm{~V}]$ \\
RD & $16.81[\mathrm{~V}]$ & $17[\mathrm{~V}]$ \\
ABD & $17.8[\mathrm{~V}]$ & $18[\mathrm{~V}]$ \\
DD & $24.06[\mathrm{~V}]$ & $24[\mathrm{~V}]$ \\
\hline
\end{tabular}

Finally, we tested the driving circuit by creating standard square signal. Figure.6 shows the wave form created by integrated circuit at different frequencies. 


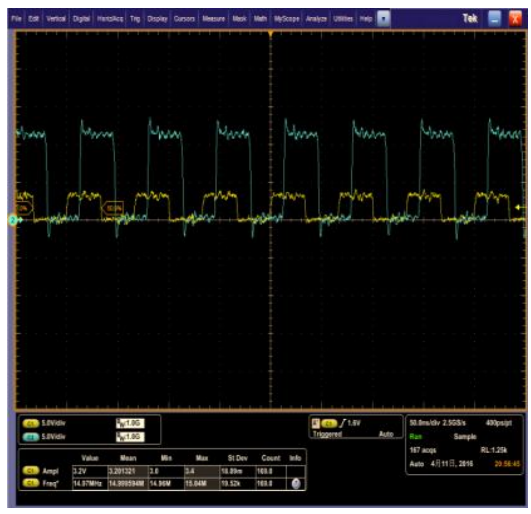

a. $15 \mathrm{MHz}$

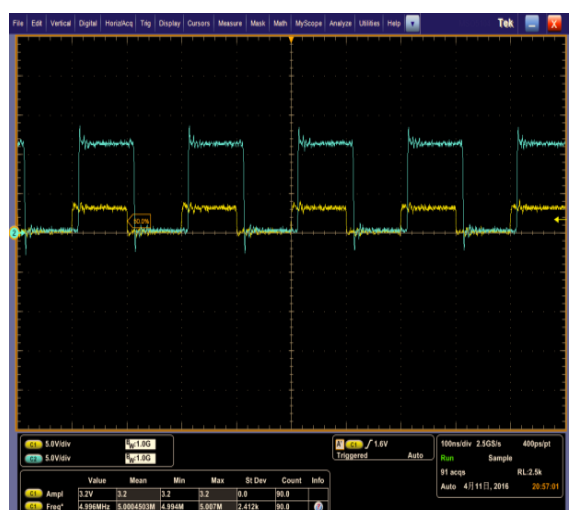

b. $5 \mathrm{MHz}$ Fig. 6 Wave form at different frequencies Fig. 7 shows the program-EM signal at different value

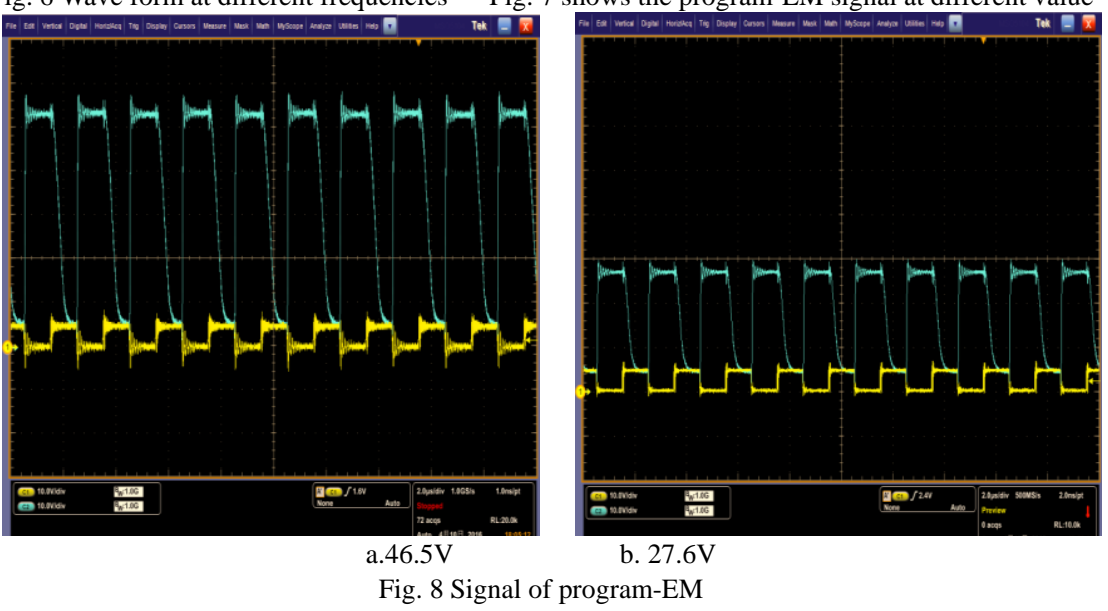

\section{Conclusion}

This paper designed a new type of CCD201 drive circuit, not only can achieve at least $15 \mathrm{MHz}$ readout frequency (CCD201 sensor output frame rate of up to 15 frames), but also ensuring the stable actual output waveform. Besides, the bias voltage can be adjusted more precisely by change the value of resistor. It makes the bias voltage circuit more flexible and practicability. The contrivance of driving circuit is proved to be feasible, and it can be used in other CCD driving circuit. 


\section{References}

[1] Yang S, Guo M, Li B, et al. Design of High Resolution High Sensitivity EMCCD Camera[C]. 2012 International Conference on Intelligent System Design and Engineering Application. IEEE Computer Society, 2012:1212-1216.

[2] Zhang W W, Chen Q. Signal-to-Noise Ratio Performance Comparison of Electron Multiplying CCD and Intensified CCD Detectors[C]. 2009 International Conference on Image Analysis and Signal Processing. 2009:337-341.

[3] Wang M F, Yang S H, Qin-Zhang W U, et al. A Driving Circuit Design for High Frame Rate EMCCD [J]. Opto-Electronic Engineering, 2011, 38(4):135-140.

[4] Sun J, Zhang B P, Cao R X. Design of EMCCD-based Driver Circuit [J]. Modern Electronics Technique, 2011.

[5] Han L, Xiong P. The analysis of operating principle and performance of EMCCD [J]. Sensor World, 2009.

[6] "The Use of Multiplication Gain in L3Vision," technical memo, E2V Technologies, 2004.).

[7] e2v technologies inc. CCD201-20 Back Illuminated 2-Phase IMO Series Electron Multiplying CCD Sensor[OL]. http://www.e2v.com 2005.06. 